\title{
Inter-relações entre o desempenho no processo de aprendizagem escolar e o desenvolvimento das capacidades motoras: revisão da literatura
}

\section{Interrelationships between performance in school learning process and the development of motor skills: a literature review}

\author{
Alessandra Antunes Tavares ${ }^{1}$, Ana Amélia Cardoso ${ }^{2}$
}

http://dx.doi.org/10.11606/issn.2238-6149.v27i1p88-93

Tavares AA, Cardoso AA. Inter-relações entre o desempenho no processo de aprendizagem escolar e o desenvolvimento das capacidades motoras: revisão da literatura. Rev Ter Ocup Univ São Paulo. 2016 jan.-abr.;27(1):88-93.

RESUMO: Esta revisão da literatura teve como objetivo identificar as principais relações existentes entre as dificuldades de aprendizagem (DA) e déficits no desenvolvimento motor, assim como analisar os principais déficits motores associados a tal condição. Realizou-se busca eletrônica nas principais bases de dados (Lilacs, Medline/ Pubmed, Bireme, Portal Capes, SciELO), com artigos publicados entre 2006 e 2014, cuja amostra contemplasse crianças em idade escolar, matriculadas na rede de ensino, com histórico de baixo desempenho escolar. Os artigos apontaram evidências da relação entre as DA e os impactos no desenvolvimento motor, visto que crianças com DA geralmente apresentam pobres habilidades motoras em comparação com seus pares com desenvolvimento típico. Conclui-se a necessidade de avaliar não somente as dificuldades específicas e funções neurológicas ligadas à aprendizagem, mas também os aspectos motores envolvidos no desenvolvimento da criança em idade escolar. Tais percepções são eficazes na elaboração de planos de intervenção preventivos/reabilitadores, reduzindo os impactos de tais dificuldades no processo de aprendizagem, auxiliando assim no melhor desempenho escolar.

DESCRITORES: Criança; Transtornos de aprendizagem; Atividade motora; Destreza motora; Desempenho psicomotor; Transtorno das habilidades motoras; Literatura de revisão como assunto.
Tavares AA, Cardoso AA. Interrelationships between performance in school learning process and the development of motor skills: a literature review. Rev Ter Ocup Univ São Paulo. 2016 Jan.Apr:;27(1):88-93.

ABSTRACT: This literature review aimed to identify the main relationships between the learning difficulties (LD) and deficits in motor development, as well as analyze the main motor deficits associated with this condition. We conducted a survey of the available evidences by electronic search at the main databases (Lilacs, Medline/Pubmed, Bireme, Portal Capes, SciELO), with articles published between 2006 and 2014 whose sample contemplate school children enrolled in the school system, with a history of poor school performance. The articles provide evidences of the relation between the LD and the impacts on the engine development, since children with LD generally have poor motor skills compared to their typically developing peers. The conclusion is the need to evaluate not only the specific difficulties and neurological functions related to learning, but also the motor aspects involved in the development of children of school age. Such perceptions are effective in developing preventive intervention plans / rehabilitators, reducing the impacts of such difficulties in the learning process, helping a better school performance.

KEYWORDS: Child; Learning disorders; Motor activity; Motor skills; Psychomotor performance; Motor skills disorders; Review literature as topic.

Este artigo é parte do Trabalho de Conclusão de Curso da primeira autora, Curso de Pós Graduação em Terapia Ocupacional - Ênfase na Saúde da Criança e do Adolescente, Faculdade de Ciências Médicas de Minas Gerais - FCMMG.

1. Curso de Pós Graduação em Terapia Ocupacional - Ênfase na Saúde da Criança e do Adolescente, Faculdade de Ciências Médicas de Minas Gerais - FCMMG, Belo Horizonte, Minas Gerais, Brasil, E-mail: tavares_ale@hotmail.com

2. Doutora em Ciências da Reabilitação pela UFMG, Departamento de Terapia Ocupacional - DTO, Universidade Federal de Minas Gerais - UFMG, Belo Horizonte, MG, Brasil, E-mail: anaameliato@yahoo.com.br

Endereço para correspondência: Alessandra Antunes Tavares. Rua Mário Martins, 315, Pompéia. CEP: 30280-450, Belo Horizonte, MG, Brasil. Telefone: (31) 98761-2426 / (31) 99791-8051.tavares_ale@hotmail.com 


\section{INTRODUÇÃO}

A s dificuldades de aprendizagem (DA) são consideradas problemas neurológicos que afetam a capacidade do cérebro para compreender, recordar ou comunicar informações, sendo também entendidas como barreiras vivenciadas pelas crianças durante a escolarização, referentes à captação ou assimilação dos conteúdos aprendidos ${ }^{1}$.

Tais dificuldades podem ser definidas como um conjunto de diferentes desordens, as quais se manifestam por déficits na aquisição e utilização da compreensão auditiva, da leitura, da fala, da escrita e/ou do raciocínio matemático ${ }^{2-3}$. Desta forma, as DA podem não estar associadas a nenhum tipo de comprometimento neurológico como atrasos mentais, doenças congênitas, deficiência física, no entanto têm impacto no desempenho da criança em diferentes atividades ${ }^{3}$. Concomitantemente não se encontra na literatura um consenso sobre uma etiologia pontual e clara para as DA, evidenciando que as mesmas apresentam múltiplos fatores que contribuem para seu surgimento ${ }^{4}$.

Almeida $^{5}$ aponta que o quadro estatístico mundial evidencia que aproximadamente $15 \%$ a $30 \%$ das crianças em idade escolar apresentam DA, sendo estas mais frequentes no sexo masculino (quatro a cinco meninos para cada menina). Alguns comportamentos são frequentemente observados em crianças com DA, por exemplo: fraco alcance da atenção, hiperatividade, dificuldade para seguir instruções, imaturidade social, dificuldade com a conversação, inflexibilidade, fraco planejamento e habilidades organizacionais, distração, falta de controle dos impulsos e falta de destreza ${ }^{1}$.

Além dos processos de aprendizagem escolar, durante o período de escolarização (entre os 06 e 12 anos), ocorre o amadurecimento das principais habilidades motoras. Segundo Okuda et al. ${ }^{6}$, cerca de $50 \%$ dos escolares com DA são identificados também com uma desordem no desenvolvimento da coordenação motora.

As alterações do desenvolvimento motor são caracterizadas por deficiências evidenciadas em diferentes áreas, tais como: coordenação motora fina, coordenação motora grossa, esquema corporal, organização espaço temporal e equilíbrio 7 . De acordo com aAssociaçãoAmericana de Psiquiatria ${ }^{8}$, a prevalência de dificuldades motoras em crianças em idade escolar varia de $6 \%$ a $8 \%$. Destes, cerca de $30 \%$ a $50 \%$ também apresentam algum tipo de DA como comorbidade ${ }^{4}$. Diferentes pesquisas reforçam a inter-relação existente entre o desenvolvimento das capacidades motoras e o desempenho no processo de aprendizagem escolar. Os componentes das habilidades motoras exercem influências na aquisição de habilidade de aprendizagem cognitiva, e estas habilidades serão utilizadas posteriormente nos processos de assimilação da leitura e escrita9-10.

Diante da presença de DA, há maior probabilidade das funções práxicas e gnósicas sofrerem alterações, comprometendo assim a destreza, a velocidade de manipulação dos objetos, refinamento do movimento, habilidades de escrita, posicionamento do corpo e da mão e consequente impacto nas tarefas funcionais ${ }^{11}$. Corroborando com tais apontamentos, Medina-Papst e Marques ${ }^{3}$, relatam atrasos no desenvolvimento motor e mudanças no controle do movimento associados às DA em escolares.

Compreender a relação entre as DA e os problemas no desenvolvimento motor de escolares pode ser uma forma eficaz para auxiliar na elaboração de planos de intervenção tanto reabilitadores quanto preventivos destas crianças. Esta revisão da literatura teve como objetivo identificar quais as principais relações existentes entre as DA e déficits no desenvolvimento motor, assim como analisar os principais déficits motores associados a tal condição.

\section{MÉTODO}

Foi realizada busca bibliográfica nas seguintes bases eletrônicas: Lilacs, Medline/Pubmed, Bireme, Portal Capes, SciELO, usando as seguintes palavraschave e suas respectivas traduções em inglês e espanhol: criança, transtornos de aprendizagem, atividade motora, destreza motora, desempenho psicomotor, transtorno das habilidades motoras.

Foram seguidos os seguintes critérios de inclusão: artigos com desfecho nas DA e déficits motores, publicados no período de 2006 a 2014, cuja amostra contemplasse crianças em idade escolar, matriculadas na rede de ensino, com histórico de baixo desempenho escolar.

Foram excluídos da revisão: estudos que abordassem crianças com alguma deficiência (intelectual ou motora, como paralisia cerebral, distrofia muscular), faixa etária adulta, artigos de revisão, dissertações e teses; trabalhos que não deixaram claros os cuidados éticos para realização dos mesmos.

Inicialmente foram selecionados 13 artigos que cumpriram os critérios acima. Durante a leitura do resumo, porém, foram descartados seis estudos, pois estes envolviam processos de intervenção direcionados as crianças com DA associados a déficits motores, sem mencionar as relações existentes. Após a leitura dos resumos, sete artigos foram incluídos. 


\section{RESULTADOS}

Os sete artigos selecionados avaliaram, caracterizaram e/ou compararam o desenvolvimento motor de crianças com
DA em relação a crianças sem dificuldades escolares. Os estudos envolveram crianças matriculadas no ensino regular, na faixa etária compreendida entre 06 e 11 anos. Na Tabela 1 encontra-se um resumo de cada estudo localizado.

Tabela 1 - Resumo das pesquisas abordando relação entre as DA e os problemas no desenvolvimento motor

\begin{tabular}{|c|c|c|c|}
\hline Autor/Ano & Título & Metodologia & Principais achados \\
\hline $\begin{array}{l}\text { Fernani et } \\
\text { al. }{ }^{7}\end{array}$ & $\begin{array}{l}\text { Motor intervention in } \\
\text { children with school } \\
\text { learning difficulties. }\end{array}$ & $\begin{array}{l}\text { População: } 28 \text { crianças entre } 06 \text { e } 11 \\
\text { anos com dificuldade de aprendizagem } \\
\text { e características de atraso no } \\
\text { desenvolvimento motor. } \\
\text { Crianças foram avaliadas antes e após } \\
\text { a aplicação de programa de intervenção } \\
\text { motora de seis meses, com frequência de } \\
\text { uma vez por semana durante uma hora. }\end{array}$ & $\begin{array}{l}\text { Mudanças nas respostas motoras foram } \\
\text { encontradas em crianças com diagnósticos como } \\
\text { TDAH, autismo, dislexia. } \\
\text { Maioria das crianças com dificuldade de } \\
\text { aprendizagem apresentou desenvolvimento } \\
\text { motor classificadas como "normal baixo". } \\
\text { Crianças com DA, antes do processo de } \\
\text { intervenção, apresentaram maiores impactos } \\
\text { em áreas relacionadas ao esquema corporal, } \\
\text { organização temporal e espacial. }\end{array}$ \\
\hline
\end{tabular}

Motor and learning $\begin{aligned} \text { Silva et al. } & \begin{array}{l}\text { disabilities in school } \\ \text { children with low } \\ \text { academic performance. }\end{array}\end{aligned}$

$\begin{array}{ll}\text { Silva et al. } & \begin{array}{l}\text { disabilities in school } \\ \text { children with low } \\ \text { academic performance. }\end{array}\end{array}$

$\begin{array}{ll}\text { Silva et al. }{ }^{2} & \begin{array}{l}\text { disabilities in school } \\ \text { children with low } \\ \text { academic performance. }\end{array}\end{array}$

População: 19 estudantes (08 meninos e 11 meninas), com média de idade de 10,3 anos.

Objetivo: verificar as dificuldades motoras

e da aprendizagem em escolares com baixo desempenho escolar.
Não foram encontradas associações entre as DA e os sexos, nem foram evidenciadas diferenças estatisticamente significativas ao comparar o desempenho motor das crianças com e sem indicativo de dificuldade de aprendizagem.
População: 406 escolares com idade entre

Beltrame; Silva ${ }^{20}$

Desempenho Motor e DA em Escolares com idade entre 7 e 10 anos.
07 e 10 anos ( 231 meninas e 175 meninos).

Comparou-se o desempenho motor de crianças com e sem dificuldade de aprendizagem.
Melhor desempenho no equilíbrio e na destreza manual nos escolares sem dificuldade de aprendizagem.

Entre os meninos, associação significativa entre as DA e o indicativo de dificuldades motoras. Meninos com DA em escritura e leitura apresentaram piores resultados na capacidade de equilíbrio.

Participantes: 22 escolares do gênero masculino distribuídos em: GI (11

Função motora fina, sensorial e perceptiva de escolares com transtorno do déficit de atenção com hiperatividade.

Okuda

et al. ${ }^{11}$

Coordenação motora fina Okuda de escolares com dislexia et al. ${ }^{6} \quad$ e transtorno do déficit de atenção e hiperatividade. escolares com TDAH) e GII (11 escolares com bom desempenho acadêmico e sem alterações de comportamento). Objetivo: caracterizar e comparar as funções motoras finas, sensorial e perceptiva de escolares com TDAH e escolares com bom desempenho escolar sem alterações do comportamento.

Participantes: 22 escolares, de ambos os gêneros, entre 06 e 11 anos, distribuídos em GI (11 escolares com TDAH) e GII (11 escolares com Dislexia).

Objetivo: descrever e comparar o desempenho da coordenação motora fina em escolares com TDAH e Dislexia.
Crianças com TDAH apresentaram, nas provas avaliadas, desempenho motor muito inferior ao desempenho de crianças sem DA.

Em uma segunda avaliação, observou-se que os alunos com TDAH obtiveram menor pontuação nas provas de Função Motora Fina, Função Sensorial e Função Perceptiva. $100 \%$ dos escolares com TDAH apresentaram disgrafia e $100 \%$ dos escolares sem DA não apresentaram qualquer alteração da escrita.

Tanto escolares com dislexia quanto escolares com TDAH apresentaram dificuldades na realização de atividades motoras finas, revelando que a idade motora fina destes escolares era inferior à idade cronológica.

Os dois grupos também apresentaram dificuldades em atividades de preensão e pressão de objetos, assim como coordenação visuoespacial. 
Tabela 1 - Continuação

\begin{tabular}{|c|c|c|c|}
\hline Autor/Ano & Título & Metodologia & Principais achados \\
\hline $\begin{array}{l}\text { Medina- } \\
\text { Papst; } \\
\text { Marques }^{3}\end{array}$ & $\begin{array}{l}\text { Avaliação do } \\
\text { Desenvolvimento Motor } \\
\text { de Crianças com DA. }\end{array}$ & $\begin{array}{l}\text { Participantes: } 30 \text { crianças ( } 21 \text { meninos e } 09 \\
\text { meninas), de } 8 \text { a } 10 \text { anos, com dificuldade } \\
\text { de aprendizagem. } \\
\text { Objetivo: investigar se crianças } \\
\text { com dificuldade de aprendizagem } \\
\text { apresentam comprometimento motor no } \\
\text { desenvolvimento dos componentes da } \\
\text { motricidade. }\end{array}$ & $\begin{array}{l}\text { Todos os grupos de crianças com DA } \\
\text { apresentaram atrasos no desenvolvimento motor. } \\
\text { Crianças com dificuldade de aprendizagem } \\
\text { apresentaram comprometimento motor } \\
\text { no desenvolvimento dos componentes } \\
\text { da motricidade, particularmente aqueles } \\
\text { relacionados às noções corporais, espaciais e } \\
\text { temporais, assim como equilíbrio. Neste caso, } \\
\text { as crianças mais velhas apresentaram maior } \\
\text { diferença entre a Idade Cronológica e a Idade } \\
\text { motora Global. }\end{array}$ \\
\hline Neto et al. ${ }^{21}$ & $\begin{array}{l}\text { Desenvolvimento } \\
\text { Motor de Crianças } \\
\text { com Indicadores } \\
\text { de Dificuldades na } \\
\text { Aprendizagem Escolar. }\end{array}$ & $\begin{array}{l}\text { Participantes: } 31 \text { crianças matriculadas de } \\
\text { 1a a 4a série com indicativo de dificuldade } \\
\text { de aprendizagem. } \\
\text { Objetivo: avaliar o desenvolvimento motor } \\
\text { de crianças com indicativo de dificuldade } \\
\text { de aprendizagem escolar. }\end{array}$ & $\begin{array}{l}\text { Associação positiva entre as DA e os atrasos } \\
\text { motores. } \\
\text { Identificação de déficit significativo entre a } \\
\text { idade cronológica e a idade motora geral; } \\
\text { Maiores déficits encontrados na área de esquema } \\
\text { corporal, organização espacial e temporal e } \\
\text { equilíbrio, assim como presença de desvios de } \\
\text { lateralidade. } \\
\text { Quanto ao desenvolvimento motor, não foram } \\
\text { identificadas diferenças entre os sexos. }\end{array}$ \\
\hline
\end{tabular}

\section{DISCUSSÃO}

Dos sete artigos analisados, seis apontaram evidências de relação entre as DA e os impactos no desenvolvimento motor. Crianças com DA geralmente apresentam pobres habilidades motoras em comparação com seus pares com desenvolvimento típico, o que indica uma associação positiva entre os aspectos.

Achados envolvendo a associação entre os problemas motores e as dificuldades relacionadas à aprendizagem em leitura foram verificados em meninas, assim como déficits relacionados ao esquema corporal ${ }^{2,12}$. Além disso, observou-se que meninas que apresentaram dificuldades na leitura evidenciaram pior desempenho motor. Outro dado importante é que meninos com DA em escrita e leitura apresentam resultados inferiores relacionados ao equilíbrio ${ }^{2,12}$.

As dificuldades na escrita e leitura são mais frequentemente associadas aos problemas na coordenação motora, e as DA em matemática estão mais relacionadas às dificuldades perceptivo-motoras (organização espaço temporal e lateralidade) $)^{2}$. Tais informações vão de encontro ao exposto por Fávero e Calsa ${ }^{13}$, onde se observa que crianças que apresentam melhor desenvolvimento motor manifestam maior facilidade na aprendizagem da leitura e da escrita.
Okuda et al. ${ }^{6}$ também observaram que a idade motora fina de escolares com Dislexia e Transtorno do Déficit de Atenção e Hiperatividade (TDAH) era inferior à idade cronológica. Verificou-se que estes escolares apresentavam dificuldades significativas em diferentes atividades que envolviam preensão e pressão de objetos (como fazer bolinha de papel, circulo com o polegar, lançar e agarrar bola, labirinto), assim como coordenação visuo-espacial. Todas estas habilidades, que exigem elevada destreza e coordenação motora, tornam-se fundamentais para a aquisição do grafismo. Em outro estudo, Okuda et al. ${ }^{11}$ verificaram a presença de disgrafia em $100 \%$ dos escolares com TDAH avaliados quando comparados a escolares sem DA, os quais não apresentaram alterações na escrita. Além disso, foram evidenciados déficits na coordenação motora fina, perceptiva (abotoar, traçar, dar laço) e sensorial (como posição da mão, tato, estereognosia, etc).

Rodrigues et al. ${ }^{14}$, ao realizar um estudo envolvendo a caligrafia, apontam que aspectos como o controle motor fino, lateralização, organização espaço-temporal, planejamento motor, integração visuo-motora, consciência sensorial dos dedos, quando alterados, levam a problemas na caligrafia (redução na habilidade da escrita), os quais comprometem o desempenho acadêmico. Corroborando com tal apontamento, Fávero e Calsa ${ }^{13}$ reforçam que o aprimoramento dos aspectos motores que estão envolvidos 
na escrita não é obtido somente por meio da execução sistematizada de exercícios ou treinos de ortografia, sendo também fundamentais as atividades espontâneas, as quais contribuem para potencializar e aprimorar o controle dos instrumentos da escrita, estimulando a aquisição das habilidades anteriormente apresentadas.

Foi observado que escolares com DA apresentam atraso motor diante da idade cronológica ${ }^{3,6,12,13}$. Frequentemente são verificados maiores déficits em áreas como esquema corporal, organização espacial e organização temporal ${ }^{3,7,12,15,16}$. Medina-Papst e Marques $^{3}$ também observaram que as maiores discrepâncias entre a idade cronológica e o desenvolvimento dos componentes da motricidade encontravam-se no grupo de crianças mais velhas (9 e 10 anos), no qual estavam presentes as maiores diferenças, evidenciando que quanto maior a idade, maiores os atrasos dos componentes avaliados.

Componentes da aprendizagem motora influenciam significativamente a aquisição de habilidade de aprendizagem cognitiva (principalmente na noção corporal, de espaço e tempo), visto que estas capacidades serão posteriormente requisitadas no processo de aprendizagem da leitura e escrita $^{9,10}$. Nas fases iniciais do desenvolvimento motor, alguns componentes são essenciais para que se adquiram padrões fundamentais de movimento, como consciência corporal, direcional e espacial, assim como ritmo, automatização, sequência de movimento e sincronia ${ }^{9,10,17}$.

Atrasos motores frequentemente associamse a outros aspectos, como prejuízos de ordem social, psicológica, os quais podem impactar o desempenho escolar $^{18}$. Logo, o envolvimento em atividades motoras e a exploração de movimentos é uma maneira positiva de aumentar o repertório motor e reforçar as habilidades que são essenciais ao raciocínio e a aprendizagem dos conceitos acadêmicos ${ }^{4,10}$.

Verifica-se que a observação detalhada e atenta dos padrões motores torna-se essencial para prevenir/ minimizar ou excluir fatores que podem impactar no aprendizado da criança ${ }^{3}$. Diante disso, a escola deve ser preparada para não dissociar o aspecto motor do

\section{REFERÊNCIAS}

1. Smith CR, Strick LW. Dificuldades de Aprendizagem de A a Z: um guia completo para pais e educadores. Porto Alegre: Artmed; 2001. desenvolvimento dos outros aspectos envolvidos na aprendizagem ${ }^{13}$. Proporcionar à criança um ambiente rico em estímulos e vivências corporais direcionadas é fundamental para a potencialização das capacidades motoras, físicas, cognitivas, sociais e afetivas 9 .

Atualmente, estudos envolvendo a análise entre as relações específicas entre habilidades motoras e de desempenho escolar em crianças com dificuldade de aprendizagem são limitados, sendo muitas vezes restritas à leitura ${ }^{19}$, o que reforça a necessidade de investigações com o foco na relação entre os diferentes subgrupos de habilidades motoras e os diferentes domínios de desempenho acadêmico. Estas percepções levariam a estratégias de avaliação e intervenção mais direcionadas a cada necessidade.

\section{CONSIDERAÇÕES FINAIS}

Compreender as relações entre as DA e o desenvolvimento motor é fundamental para orientar professores e educadores e auxiliá-los na identificação, manejo e elaboração de estratégias - dentro do ambiente escolar - de forma a estimular o melhor desempenho de cada criança, tendo em vista que as dificuldades apresentadas por elas são únicas, merecendo atenção especial e direcionada. Tal compreensão torna-se também importante para o desenvolvimento de programas que não somente identifiquem tais sinais (DA associados a questões motoras), mas também utilizem da própria psicomotricidade como forma de prevenir/ reduzir as consequências das DA no rendimento do escolar e adaptação da criança neste ambiente.

Assim, ampliar a percepção diante das DA - indo além do aspecto cognitivo, e considerando múltiplos fatores, como as relações existentes entre as habilidades motoras - é fundamental para que escola, família, comunidade compreendam que são necessárias articulações entre os diferentes parceiros envolvidos neste processo (profissionais da saúde, professores, educadores, família) na busca das melhores estratégias a serem implementadas na tentativa de prevenir maiores agravos e intervir nas necessidades já existentes.

2. Silva J, Beltrame TS, Oliveira AVP, Sperandio FF. Dificuldades motoras e de aprendizagem em crianças com baixo desempenho escolar. Rev Bras Crescimento 
Desenvolv Hum. 2012;22:41-6. http://dx.doi.org/10.1590/ S0104-12822012000100006.

3. Medina-Papst J, Marques I. Avaliação do desenvolvimento motor de crianças com dificuldades de aprendizagem. Rev Bras Cineantropom Desempenho Hum. 2010;12:36-42. http://dx.doi.org/10.5007/1980-0037.2010v12n1p36.

4. Prestes D, Weiss S, Araújo J. A equoterapia no desenvolvimento motor e autopercepção de escolares com dificuldade de aprendizagem. Cien Cogn. 2010;15:192-203. Disponível em: http://pepsic.bvsalud.org/pdf/cc/v15n3/ v15n3a16.pdf

5. Almeida R. As dificuldades de aprendizagem: repensando o olhar e a prática no cotidiano da sala de aula [Dissertação]. Florianópolis: Universidade Federal de Santa Catarina; 2002. Disponível em: https://repositorio.ufsc.br/bitstream/ handle/123456789/84199/187613.pdf?sequence=1.

6. Okuda PMMO, Lourencetti MD, Santos LCA, Padula NAMR, Capellini SA. Coordenação motora fina de Escolares com dislexia e Transtorno do déficit de Atenção e Hiperatividade. Rev CEFAC. 2011;13:876-85. http://dx.doi. org/10.1590/S1516-18462011005000048.

7. Fernani DCGL, Prado MTA, Fell RF, Reis NL, Bofi TC, Ribeiro EB, et al. Motor intervention in children with school learning difficulties. J Hum Growth Develop. 2013;23:20914. http://dx.doi.org/10.7322/jhgd.61301.

8. Associação Americana de Psiquiatria (APA). Manual diagnóstico e estatístico de transtornos mentais. 4a ed. Porto Alegre: Artes Médicas; 1995.

9. Amaro K. Intervenção motora para escolares com dificuldade de aprendizagem [Dissertação]. Florianópolis: Universidade do Estado de Santa Catarina; 2010. Disponível em: http:/www.motricidade.com.br/pdfs/edm/dis.1.pdf.

10. Medina J, Rosa G, Marques I. Desenvolvimento da organização temporal de crianças com dificuldades de aprendizagem. Rev Educ Física UEM (Maringá). 2006;17:107-116. http://dx.doi.org/10.4025/ reveducfisv $17 \mathrm{n} 1 \mathrm{p} 107-116$.

11. Okuda PMM, Pinheiro FH, Germano GD, Padula NAMR, Lourencetti MD, et al. Função motora fina, sensorial e perceptiva de escolares com transtorno do déficit de atenção com hiperatividade. J Soc Bras Fonoaudiol. 2011;23:351-7. http://dx.doi.org/10.1590/S2179-64912011000400010.

12. Piucco EC, Neto FR, Almeida GMF, Caon G, Caram JA, Ribeiro J. Desenvolvimento motor de crianças com indicadores de dificuldades na aprendizagem escolar. Rev Bras Cien Mov. 2007;15:45-51. Disponível em: http://portalrevistas.ucb.br/index.php/RBCM/article/ view/729/732.

13. Fávero M, Calsa G. Desenvolvimento Psicomotor e Aprendizagem da Escrita [Dissertação]. Maringá: Universidade Estadual de Maringá; 2004. Disponível em: http://www.ppe.uem.br/dissertacoes/2005-Maria_Teresa. pdf.

14. Rodrigues S, Castro M, Ciasca S. Relação entre indícios de disgrafia funcional e desempenho acadêmico. Rev CEFAC. 2009;11:221-7. http://dx.doi.org/10.1590/S151618462008005000005

15. Barreto SJ. Psicomotricidade: educação e reeducação. 2a ed. rev. e ampl. Blumenau: Acadêmica Publicações; 2000.

16. Ferreira J. Saúde escolar: aspectos biopsicossociais em crianças com dificuldade de aprendizagem [Dissertação]. Florianópolis: Universidade do Estado de Santa Catarina; 2007. Disponível em: http://www.tede.udesc.br/tde_busca/ arquivo.php? codArquivo $=1050$.

17. Prestes DB, Weiss S, Araújo JCO. A equoterapia no desenvolvimento motor e autopercepção de escolares com dificuldade de aprendizagem. Cien Cogn. 2010;15(3):192203. Disponível em: http://pepsic.bvsalud.org/pdf/cc/ v15n3/v15n3a16.pdf.

18. Willrich A, Azevedo C, Fernandes J. Desenvolvimento motor na infância: influência dos fatores de risco e programas de intervenção. Rev Neurocienc. 2009;17:51-6. Disponível em: http://services.epm.br/dneuro/neurociencias/226 revisao.pdf.

19. Westendorp M, Hartman E, Houwen S, Smith J, Visscher C. The relationship between gross motor skills and academic achievement in children with learning disabilities. Res Develop Disabil. 2011;32:2773-9. http://dx.doi. org/10.1016/j.ridd.2011.05.032.

20. Beltrame T, Silva J. Desempenho motor e DA em escolares com idade entre 7 e 10 anos. Rev Motricidade. 2011;7(2):57-68. http://dx.doi.org/10.6063/ motricidade.7(2).111.

21. Rosa Neto F, Almeida GMF, Caon G, Ribeiro J, Caram JA, PiuccoEC.Desenvolvimentomotor de crianças comindicadores de dificuldades na aprendizagem escolar. Rev Bras Cien Mov. 2007;15(1):45-51. Disponível em: http://portalrevistas.ucb.br/ index.php/RBCM/article/viewFile/729/732. 